\title{
¿Son los nuevos criterios de evaluación en el marco del EEES adecuados para valorar el rendimiento académico de los alumnos? Experiencia en Contabilidad Financiera
}

Are the new EHEA assessment methods appropriate to evaluate the academic performance of students? An experience in Financial Accounting

David Pascual Ezama (david.pascual@ccee.ucm.es)

Ma Mar Camacho Miñano (marcamacho@ccee.ucm.es)

Elena Urquía Grande (eurquiag@ccee.ucm.es)

\author{
Alexander Müller (alexander.muller@ccee.ucm.es) \\ Universidad Complutense de Madrid (España) \\ http://dx.doi.org/10.12795/EDUCADE.2011.i02.05
}

\begin{abstract}
RESUMEN: La Facultad de Ciencias Económicas y Empresariales de la Universidad Complutense de Madrid ha puesto en marcha durante el curso 2009-2010 la implantación de los nuevos Grados en Administración y Dirección de Empresas y en Economía, ajustándose a los nuevos criterios recogidos en el marco del Espacio Europeo de Educación Superior (EEES). Una de las novedades es la evaluación acumulativa por actividades frente a la evaluación tradicional. Profesores de Contabilidad Financiera de primer curso han aplicado la metodología de enseñanza activa y su consecuente método de evaluación. Los resultados obtenidos en este trabajo empírico cuestionan este método de evaluación y promueve mejoras alternativas para implementar otros tipos de evaluación, además de que podría ser extrapolable a otras materias. Todo ello promueve una mejora continua en la docencia universitaria y un aprendizaje significativo del alumno.
\end{abstract}

PALABRAS CLAVE: EEES, Contabilidad Financiera, Metodología docente activa, Evaluación acumulativa por actividades, Rendimiento académico.

ABSTRACT: The Economics and Business Administration School of the Complutense University of Madrid has launched during the 2009-2010 academic year the new degrees in Economics and in Business Administration. These degrees have adjusted to the new criteria established in the European High Education Area (EHEA) framework. A new characteristic is the student cumulative activities assessment opposite to the traditional way of assessment. Three Financial Accounting lecturers have designed and applied, in the first year degree, active teaching methodologies and its consequent evaluation. The results of this empirical experiment show it is not the optimal assessment method and suggests other alternative methodologies could suit best. All of this together motivates a continuous improvement in the university teaching process and a deep learning for the students.

KEYWORDS: EHEA, Financial Accounting, active teaching methodology, Cumulative activities assessment, Academic performance.

Artículo. Recibido: 18-01-11 - Versiones revisadas: 23-03-11, Aceptado: 30-06-11

Licencia Creative Commons (CC) BY-NC-ND · Asociación Española de Contabilidad y Administración de Empresas - AECA 


\section{INTRODUCCIÓN}

El conocimiento y las capacidades profesionales que necesita actualmente el mercado de trabajo están en consonancia con el desarrollo tecnológico en la denominada sociedad de la información, que se caracteriza por continuos y rápidos cambios. Hace unas décadas las empresas demandaban profesionales con amplios conocimientos que, junto a la experiencia en el puesto de trabajo, garantizaba un buen desarrollo de su actividad en el tiempo. Hoy en día las empresas demandan profesionales con capacidad crítica y con las competencias y habilidades suficientes para poder adaptarse a un entorno económico cambiante. Así, el sistema educativo en cualquier país debe proporcionar el aprendizaje adaptado a los requerimientos de la sociedad.

La Facultad de Ciencias Económicas y Empresariales de la Universidad Complutense de Madrid (UCM) ha puesto en marcha durante el curso 2009-2010 la implantación de los nuevos Grados en Administración y Dirección de Empresas (GADE) y en Economía (GECO), ajustándose a los nuevos criterios recogidos en el marco del Espacio Europeo de Educación Superior (EEES). Esta implantación ha supuesto una oportunidad para introducir innovaciones educativas fundamentadas en un cambio de metodología de enseñanza y un apoyo en las Tecnologías de la Información y Comunicación (TIC).

Por lo tanto, los docentes universitarios hemos tenido que asumir los desafíos del cambio condicionados, en muchas ocasiones, por factores externos al mismo como los presupuestos o las legislaciones oportunas. El profesor de Contabilidad ha tenido que hacer frente a los siguientes retos: por un lado, aplicar la metodología docente activa adecuada para adaptarse al EEES; por otro lado, ajustar la enseñanza de la contabilidad a la convergencia contable europea (International Financial Reporting Standards (IFRS) junto con la norteamericana (Statements of Financial Accounting Standards (SFAS)). Adicionalmente, sería recomendable que el docente incorporase en su metodología activa las TIC.

En todo este proceso de cambio consideramos que la metodología docente es clave para que los alumnos consigan un buen aprendizaje. El profesor, aplicando una metodología activa, puede conseguir que el alumnado obtenga las competencias y habilidades que la sociedad demanda. La metodología docente activa se basa en el concepto de "pedagogía emergente" centrado en el estudiante, que debe ser capaz de crear conocimiento a partir de la unión entre lo que sabe, la información que recibe y su propia experiencia personal (Vygotsky, 1962) siendo este es el punto de partida de los requerimientos de la sociedad actual (Prince, 2004). El profesor debe utilizar todas las estrategias docentes disponibles para conseguir un buen aprendizaje del estudiante, para que cuando finalice su formación y sea un profesional, sepa adaptarse a diferentes situaciones, donde pueda aplicar sus conocimientos a solucionar problemas reales con actitud crítica y sepa rebatir ideas o corrientes de pensamiento diferentes. Para conseguirlo deberá utilizar las nuevas tecnologías (como un medio y no como un fin en sí mismo) ya que facilitan el acceso a una elevada cantidad de información, entre otras ventajas.

Evidentemente, si los métodos docentes cambian y los contenidos se reajustan, la evaluación del proceso de aprendizaje del alumno no puede mantenerse siguiendo el esquema tradicional, considerando únicamente la calificación del examen final. De hecho, la evaluación regula el aprendizaje, ya que el estudio de la mayoría de los alumnos está condicionado por las demandas evaluativas del profesor (Murphy, 2006). De esta manera, la evaluación de todas las actividades propuestas en una metodología activa debe formar parte del proceso de evaluación. Además, la calificación debe ser entregada en un corto espacio de tiempo ya que se ha 
demostrado que aumenta la motivación del alumnado en el estudio (Trees y Jackson, 2007).

En este entorno de adaptación al EEES, profesores de Contabilidad Financiera del primer curso han aplicado una metodología de enseñanza activa utilizando diferentes herramientas y su consecuente método de evaluación: la evaluación acumulativa por actividades. Ésta consiste en calificar cada actividad (participación activa en el aula y en seminarios, resolución de casos y/o ejercicios, realización y presentación de trabajos individuales o en grupo, exámenes parciales, examen final) con un porcentaje para cada actividad hasta llegar al $100 \%$ de la nota final (VERIFICA UCM, 2008).

El trabajo está estructurado de la siguiente manera: primero se expone una revisión bibliográfica sobre el estado de la cuestión más allá del ámbito contable, luego se describe la investigación realizada y la metodología utilizada así como el tratamiento estadístico aplicado para llegar a una discusión de resultados y unas conclusiones. Los resultados obtenidos en este trabajo empírico, que compara la evaluación de la metodología activa con la calificación del examen final, cuestionan los actuales métodos de evaluación, pueden promover mejores alternativas para implementar otros tipos de evaluación y motivan así una mejora continua en la metodología docente y en el aprendizaje del alumno.

\section{ESTADO DE LA CUESTIÓN}

En el análisis de la situación de partida tenemos que hacer hincapié en dos ideas básicas: el tipo de metodología docente utilizada por el profesor y la evaluación elegida. Ambas cuestiones tienen, de manera directa o indirecta, impacto en la calidad del aprendizaje del alumno y deberían ser tenidas en cuenta si se quiere conseguir que se mejore la formación ofrecida por la universidad española dentro del marco del EEES.

\subsection{AlCANCE Del CAMBio en la metOdología docente}

Con el desarrollo de los grupos pilotos del EEES en todas las universidades españolas, se ha venido desarrollando en los últimos años un debate entre los docentes: si la metodología "moderna" es mejor que la clase magistral. Sin embargo, este debate no es nuevo. La metodología docente activa se empieza a aplicar en los años 70 en universidades de medicina e ingenierías en Estados Unidos en respuesta a un cambio en las demandas formativas de la sociedad americana (Yuretich et al., 2001; Prince y Prince, 2004; Felder, 2006). Años más tarde, se generaliza su uso en las demás áreas de conocimiento incluida la de Contabilidad.

Las herramientas utilizadas dentro de esta metodología docente activa son muchas y diversas. Con la idea de mejorar y añadir interacción a las clases magistrales surgió la utilización de transparencias y preguntas tipo test como complemento a los contenidos teóricos (Clinton y Kohlmeyer, 2005; Nouri y Shahid, 2005). Por otro lado está el método del caso que consiste en entregar a los alumnos la problemática de una empresa real con información desestructurada (Bonk, Smith y Stevenson, 1998; Weil et al., 2001, Cullen, Richardson y O`Brien, 2004; Duff, 2004; Springer y Faye, 2004; Arquero et al., 2007; Ballantine, Duff y McCourt, 2008). Otra herramienta muy utilizada es el método del Aprendizaje Basado en Problemas (ABP) o Problem Based Learning (PBL), con una orientación hacia un programa integrado y organizado en problemas de la vida real donde convergen las diferentes áreas del conocimiento (Schmidt, Daphnee y Patel, 1987; Albanese y Mitchell, 1993; Nandi, Chan y Chan, 2000; Milne y McConneill, 2001; Antepohl y Herzig, 2002; Gurpinar et al., 2005; Heaghy y Lehman, 2005; Lycke, Grottum y Stromso, 2006). 
Adicionalmente, en cualquiera de los trabajos de investigación sobre metodología de enseñanza activa existe un elemento común que es el de utilizar las TIC como apoyo a las clases (Bryant y Hunton, 2000; Holbert y Karady, 2009) como, por ejemplo, las simulaciones (Gordon et al., 2006; Urquía, et al., 2009); los programas informáticos interactivos diseñados "ad hoc" para la materia a enseñar (Halabi, Tuovinen y Farley, 2005; Haghighi, Vakil y Wetiba, 2006; Potter y Johnston, 2006); Savander-Ranne, Luden y Kolari, 2008), los mandos interactivos (Uhari, Renko y Soini, 2003; Camacho, 2009) y las herramientas de comunicación como foros, blogs o redes sociales (Williams y Jacobs, 2004; Greenhow, Robelia y Hughes, 2009; Chaín Martínez y Sánchez, 2010).

Otros estudios han analizado el impacto de la manera de agrupar a los alumnos en su aprendizaje. En general, el hecho de que los alumnos trabajen en equipo conlleva el uso de una metodología docente activa, ya que desde el planteamiento original del problema hasta su solución es necesario que lo hagan de manera colaborativa (Terenzini et al., 2001; Holbert y Karady, 2009) o cooperativa (Ravenscroft et al., 1995; Felder, Felder y Dietz, 1998; Tong Wu, 2005; Gabbin y Wood, 2008). En esta experiencia de aprendizaje comparten la posibilidad de practicar y desarrollar habilidades, de observar y reflexionar sobre actitudes y valores, negociar puntos de vista diferentes, que en el método convencional difícilmente podrían ponerse en acción. En estas actividades grupales los alumnos toman responsabilidades y acciones que son básicas en su proceso formativo y que deberán desarrollar en un futuro en su puesto de trabajo.

En esta misma línea, muchos estudios profundizan no solo en la metodología docente sino además en cómo ésta influye en el tipo de aprendizaje adquirido por los alumnos: superficial (surface learning) o significativo (deep learning). Éste último tipo de aprendizaje constituye uno de los objetivos de la metodología activa para que el alumno al final retenga los conceptos básicos de la asignatura en el largo plazo (Marton y Sajlö, 1976; Felder, 1995; Kvam, 2000; Boyce et al., 2001; Lord y Robertson, 2006); Tovia y Liu, 2007). Además, hay autores que analizan cómo el aprendizaje del alumno viene condicionado por variables extrínsecas como la familia, la aptitud o el ambiente de estudio y por variables intrínsecas como la motivación, el esfuerzo, las expectativas o la percepción (Wooten, 1998); Ballantine y McCourt, 2004; Del Favero et al., 2007). En concreto, diferentes trabajos de investigación se centran en la motivación del alumno que, unido a una metodología docente activa, influyen en un aprendizaje más profundo y duradero (Asbaugh, Johnstone y Warfield, 2002; Arquero, Jiménez y Joyce, 2004; Clinton y Kohlmeyer, 2005; Johnson y Dasgupta, 2005; Danzinger y Eden, 2006; Sánchez, 2009).

Otra línea de investigación analiza el impacto de la metodología docente en el desarrollo de las competencias cognitivas y transversales de los alumnos tan importantes en el marco EEES y que, a su vez, revierte en la mejora de resultados académicos y en la vida profesional (Weil et al., 2001; Arquero et al., 2004). Ahora bien, la valoración de los resultados académicos tiene que evolucionar en el mismo sentido por lo que nos planteamos la siguiente pregunta de investigación:

PI: Si se adopta una metodología docente activa ¿̇es necesario realizar un cambio en los criterios de evaluación?

\subsection{IMPACTO dE LA EVALUACIÓN EN EL RENDIMIENTO ACADÉMICO DEL ALUMNO}

El aprendizaje y la evaluación son dos conceptos íntimamente relacionados ya que la forma de evaluación condiciona el proceso de adquisición de conocimientos (Villardón y Yaniz, 2004). Además, el sistema de evaluación de las asignaturas condiciona las decisiones de los estudiantes (número de horas de estudio, uso de la biblioteca, bibliografía, reuniones...). Plantear al alumno una serie de actividades 
(trabajos, ejercicios, supuestos, etc.) que no van a ser evaluadas desmotivará la realización de las mismas.

Como se ha expuesto en el epígrafe anterior, la mayoría de los trabajos de investigación en este area han versado principalmente en la comparativa con la metodología tradicional, la descripción de las herramientas utilizadas, las variables psicológicas del alumno que influyen en el aprendizaje, la manera de agrupar a los estudiantes y el efecto que todo ello tiene en el aprendizaje significativo de los alumnos (Kopp y Phillips, 2005; Martin, Rivale y Diller, 2007). Sin embargo, los estudios sobre la manera de evaluar y su impacto en el rendimiento académico del alumno universitario son escasos, a pesar de considerarse esencial la evaluación en la actividad formativa del alumno (Molero, 2007).

De todos los tipos de evaluación existente, el marco del EEES demanda conseguir lo que algunos autores denominan "evaluación auténtica", que intenta ajustar lo que se evalúa a los objetivos de aprendizaje pretendidos (Ahumada (1998)). Con el método tradicional la evaluación consiste en analizar el resultado final del conocimiento adquirido y no en evaluar el proceso que es un aspecto básico de la metodología activa (Dochy, Seger y Dierick, 2008). La evaluación debe ser consecuente con el tipo de aprendizaje realizado. Los pilares básicos sobre los que se debe asentar la nueva evaluación docente son: en primer lugar, la evaluación debe servir de guía para concretar lo que se debe aprender y en segundo lugar, debe utilizarse para mejorar la calidad de la enseñanza ya que conlleva reflexionar sobre el proceso de aprendizaje (Zabalza, 2001; Harrington (2010). Ambos enfoques son complementarios y necesarios en la evaluación en el marco del EEES.

Existen diferentes métodos de evaluación en función de múltiples criterios: evaluación por portafolios, la evaluación reconceptualizada, la evaluación por pares, autoevaluación, coevaluación (Álvarez, 2008). El método más adecuado será aquel que evalúe el desarrollo de las competencias generales, transversales y específicas adquiridas durante el proceso formativo en cada herramienta de metodología activa utilizada. Al finalizar el proceso de aprendizaje, se debe calificar al estudiante y valorar la adquisición de competencias y conocimientos previamente programados al inicio del curso. Hay una amplia diversidad de instrumentos con los que concretar los distintos tipos de evaluación: escalas de observación, tests, entrevistas, registros, pruebas escritas y orales, diarios, anecdotarios, trabajos, construcción de aparatos $u$ objetos, Sanchez y Gairín, 2008). Por lo tanto, si las herramientas docentes para conseguir las competencias de los alumnos actuales han cambiado, los métodos de evaluación deben ajustarse a dicho cambio.

Por el contrario, la evaluación tradicional se fundamenta en el examen final. El alumno concibe el aprendizaje para aprobar más que para aprender. Este sistema de evaluación no es adecuado dentro del sistema educativo del EEES, que debe valorar los resultados conseguidos por el alumno en todo el proceso de aprendizaje a lo largo del curso, es decir, una evaluación integral (Delgado y Oliver, 2006).

El proceso de evaluación en la metodología activa consiste en valorar todas las actividades que el alumno realiza para mejorar sus competencias, sin olvidar la existencia de un examen final que englobe todos los conocimientos adquiridos. Ese examen final debería poder superarse y, si la evaluación de las actividades está bien diseñada, los resultados del examen final deberían coincidir con los resultados de la evaluación acumulativa por actividades realizada durante el curso. Sin embargo, no se encuentra evidencia empírica de que esto realmente suceda y por lo tanto se plantea la siguiente pregunta de investigación:

PI2: ¿̇El método de evaluación afecta a la calificación final obtenida por el alumno? 


\section{METODOLOGÍA}

\subsection{PARTICIPANTES}

La presente investigación se llevó a cabo con estudiantes del primer curso de la asignatura "Contabilidad Financiera", en las nuevas carreras de GADE y GECO, durante el curso académico 2009/2010. Se trata de una asignatura obligatoria cuatrimestral, que se imparte en el segundo cuatrimestre.

La muestra está formada por 127 estudiantes, todos entre 18 y 23 años, de los que un $53,5 \%$ eran mujeres. La mayoría de ellos son españoles $(83,2 \%)$ y han cursado la asignatura de Economía en el bachiller $(69,2 \%)$ por lo que tienen conocimientos previos de la materia. Tan sólo un 16,1\% trabaja mientras cursa sus estudios y sólo uno de ellos había cursado la materia de la "Contabilidad Financiera" en una carrera universitaria con anterioridad. La motivación principal que animó a los estudiantes a elegir la carrera universitaria fue: "les gustaba la economía" (36,4\%); "por las salidas profesionales" (25,4\%); "por no haber logrado la nota media necesaria para acceder a otra carrera" (16,1\%); "motivos personales o familiares" (13,6\%); "vocación" (5,1\%) y "por insatisfacción con otra carrera" $(3,4 \%)$.

\subsection{Procedimiento}

Las clases fueron impartidas en tres grupos de estudiantes por profesores distintos. El tiempo dedicado a la docencia en esta asignatura es de cuatro horas semanales, de las cuales tres se imparten al grupo completo en clase y una hora en forma de seminarios, en las que se divide al grupo en dos subgrupos para permitir unas clases más personalizadas y dinámicas. Las clases consisten en una breve introducción y discusión de los conceptos teóricos de cada tema previamente publicados en un entorno virtual de enseñanza-aprendizaje (EVEA). Esto se complementa con la realización de supuestos prácticos donde los alumnos deben participar activamente resolviéndolos colectivamente en el aula. Adicionalmente deberán resolver supuestos prácticos similares individualmente fuera del aula y entregarlos al profesor para recibir un feedback. Para complementar la metodología activa se han utilizado PowerPoint, Internet, la base de datos SABI y los mandos interactivos (EduClick). Cada uno de los profesores ha aplicado la misma metodología de docencia, ha seguido el mismo temario, ha utilizado el mismo material de clase y los mismos casos prácticos y ha aplicado el mismo sistema de evaluación. El sistema de evaluación utilizado fue el de evaluación acumulativa por actividades, conforme a la horquilla propuesta en los criterios que establece la ANECA.

En este sistema de evaluación acumulativa, la nota del examen final supone el $50 \%$ de la nota total de la asignatura. El contenido del examen final incluyó toda la materia impartida en clase, centrándose en la contabilización de las transacciones empresariales en los libros Diarios, Mayor y de Inventario y cuentas anuales, la elaboración de los estados financieros y los aspectos teórico-prácticos básicos de contabilidad financiera a nivel introductorio. Para realizar el examen final era imprescindible que los estudiantes hubieran asistido a las dos pruebas parciales. El otro $50 \%$ de la nota total se compone de la calificación de las distintas actividades que se desarrollaron en clase aplicando la metodología docente activa: exámenes parciales, trabajos realizados en los seminarios y la participación activa en clase.

Se realizaron dos exámenes parciales (cada uno con una ponderación del 5\%), que se llevaron a cabo aproximadamente al mes de comenzar la asignatura y el segundo una vez impartida la mitad de la materia, para comprobar los conocimientos adquiridos y la comprensión de los fundamentos básicos de la materia por parte de los 
estudiantes. Estas pruebas parciales por su parte se componen de una parte "tipo-test" mediante la cual se comprueban los conocimientos teóricos y otra parte de ejercicios de cálculo y de elaboración de los estados financieros básicos.

Los seminarios representan un $25 \%$ sobre la nota total. En la Tabla 1 se presenta el tema y objetivos de cada uno de los seminarios. El restante $15 \%$ de la nota total se basa en la participación activa del estudiante en clase. Dentro de este último concepto cabe resaltar la realización de pequeñas pruebas al finalizar alguna clase, la realización de ejercicios cortos, la entrega y posterior corrección de supuestos.

TAbla 1. Resumen de Seminarios realizados

\begin{tabular}{|c|c|c|}
\hline & Actividad & Objetivo \\
\hline Seminario 1 & $\begin{array}{l}\text { Búsqueda de información } \\
\text { financiero-contable en páginas } \\
\text { Web oficiales (Comisión Nacional } \\
\text { del Mercado de Valores (CNMV), } \\
\text { Registro Mercantil (RM)...etc.) }\end{array}$ & $\begin{array}{l}\text { Introducir al alumno en la búsqueda } \\
\text { de información económico- } \\
\text { financiera en Internet y aprender el } \\
\text { contenido, de manera intuitiva, de } \\
\text { los estados financieros básicos. }\end{array}$ \\
\hline Seminario 2 & $\begin{array}{l}\text { Búsqueda de información de } \\
\text { empresas reales en la base de } \\
\text { datos de empresas SABI }\end{array}$ & $\begin{array}{l}\text { Enseñar al alumno el uso en una } \\
\text { fase inicial de una base de datos } \\
\text { con información financiera } \\
\text { contable a través de criterios de } \\
\text { búsqueda }\end{array}$ \\
\hline Seminario 3 & $\begin{array}{l}\text { Lectura e interpretación de la } \\
\text { Memoria de las Cuentas Anuales } \\
\text { de una empresa real, información } \\
\text { que utilizarán en su vida laboral } \\
\text { de manera directa o indirecta. }\end{array}$ & $\begin{array}{l}\text { Acercar los conceptos básicos de } \\
\text { la Contabilidad Financiera a la } \\
\text { realidad empresarial }\end{array}$ \\
\hline Seminario 4 & $\begin{array}{l}\text { Simulación de un proyecto de } \\
\text { inversión en dos empresas reales y } \\
\text { posterior seguimiento de } \\
\text { cotización en Bolsa para } \\
\text { conseguir un rendimiento sobre la } \\
\text { inversión }\end{array}$ & $\begin{array}{l}\text { Desarrollo de la capacidad de } \\
\text { trabajo en equipo y negociación } \\
\text { para la selección y seguimiento de } \\
\text { las empresas. }\end{array}$ \\
\hline Seminario 5 & $\begin{array}{l}\text { Introducción de hechos } \\
\text { contables en el programa } \\
\text { informático A3CON, incluyendo } \\
\text { un supuesto con facturas reales }\end{array}$ & $\begin{array}{l}\text { Introducir al alumno en la llevanza } \\
\text { de la contabilidad como en } \\
\text { cualquier empresa real en la } \\
\text { actualidad }\end{array}$ \\
\hline Seminario 6 & $\begin{array}{l}\text { Presentación oral del proyecto de } \\
\text { inversión de las empresas reales } \\
\text { escogidas }\end{array}$ & $\begin{array}{l}\text { Desarrollo de la capacidad del } \\
\text { alumno de la comunicación y } \\
\text { transmisión de información en } \\
\text { público }\end{array}$ \\
\hline
\end{tabular}

En resumen, la siguiente tabla (Tabla 2 ) recoge las ponderaciones de la evaluación acumulativa por actividades. 
TAbla 2. Criterios de eValuación acumulativa por aCtividades

\begin{tabular}{|c|l|c|}
\hline \multicolumn{2}{|c|}{ Actividades realizadas y evaluadas } & Ponderación \\
\hline+ & 2 exámenes parciales & $10,0 \%$ \\
\hline+ & Nota del seminario & $25,0 \%$ \\
\hline+ & Participación activa en clase & $15,0 \%$ \\
\hline$=$ & Total evaluación acumulativa por actividades & $\mathbf{5 0 , 0 \%}$ \\
\hline+ & Calificación del examen final & $50,0 \%$ \\
\hline$=$ & Evaluación final de la asignatura & $100,0 \%$ \\
\hline
\end{tabular}

\subsection{ANÁLISIS ESTADÍSTICO}

Con el objetivo de tratar de dar respuesta a nuestras preguntas de investigación, se han calculado las diferencias entre las calificaciones obtenidas en el examen final de la asignatura para el conjunto de estudiantes y la evaluación final obtenida por el sistema de evaluación acumulativa por actividades. Se ha observado la significatividad de las diferencias encontradas mediante un análisis de medidas repetidas. Por otra parte, se han analizado las correlaciones existentes entre las calificaciones de los exámenes y la evaluación final con las características demográficas de los estudiantes para comprobar si existían diferencias en función del género, edad, nacionalidad y situación laboral. Finalmente, hemos realizado un análisis descriptivo de las calificaciones medias obtenidas por los alumnos en función de la motivación por la que seleccionaron su carrera universitaria.

\section{RESULTADOS}

En primer lugar, hay que destacar que los resultados obtenidos (calificación final) por los alumnos de la asignatura Contabilidad Financiera en los nuevos Grados correlacionan positivamente tanto con el examen final como con el segundo examen parcial. Cabe destacar que cuando se realizó este examen parcial se había impartido la mitad de los contenidos del programa docente (Tabla 3).

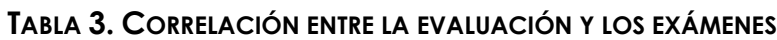

\begin{tabular}{|c|c|c|c|c|}
\hline & Calificación & $\begin{array}{c}\text { Examen } \\
\text { Farcial }\end{array}$ & $\begin{array}{c}\text { Examen } \\
\text { Final }\end{array}$ \\
\hline Calificación Final & $\begin{array}{c}\text { Correlación de } \\
\text { Pearson }\end{array}$ & 1 &, $587\left(^{* *}\right)$ &, $606\left(^{* *}\right)$ \\
\hline $\begin{array}{c}\text { Sig. (bilateral) } \\
\text { Segundo Examen } \\
\text { Parcial }\end{array}$ & $\begin{array}{c}\text { Correlación de } \\
\text { Pearson }\end{array}$ &, $587\left(^{* *}\right)$ & 1 &, 000 \\
\hline Sig. (bilateral) &, 000 &, $918\left(^{* *}\right)$ \\
\hline Examen Final & $\begin{array}{c}\text { Correlación de } \\
\text { Pearson }\end{array}$ &, $606\left(^{* *}\right)$ &, $918\left(^{* *}\right)$ & 1 \\
\hline & Sig. (bilateral) &, 000 &, 000 & \\
\hline
\end{tabular}

** La correlación es significativa al nivel 0,01 (bilateral). $\mathrm{N}=127$ 
Ahora bien, los resultados estadísticos encontrados muestran dos tendencias. Por un lado, aquellos alumnos que han obtenido una calificación en el examen final inferior a siete sobre diez consiguen mejorar su evaluación global con el esfuerzo realizado en el resto de las actividades evaluadas por el profesor (seminarios, casos, ejercicios, participación activa en clase..). Por otro lado, los alumnos que obtienen calificaciones superiores a siete en el examen final ven disminuida su evaluación global al aplicar el sistema de evaluación acumulativa por actividades (Tabla 4).

TABla 4. Diferencias entre las notas del examen final (J) y la eValuación Global (I) DE LA ASIGNATURA POR GRUPOS.

\begin{tabular}{|c|c|c|c|c|c|}
\hline \multirow{2}{*}{$\begin{array}{l}\text { Notas } \\
\text { Examen } \\
\text { Final }\end{array}$} & \multirow{2}{*}{$\begin{array}{c}\text { Diferencia } \\
\text { entre medias } \\
\quad(I-J)\end{array}$} & \multirow{2}{*}{ Error típ. } & \multirow{2}{*}{$\begin{array}{c}\text { Significación(a } \\
\text { ) }\end{array}$} & \multicolumn{2}{|c|}{$\begin{array}{c}\text { Intervalo de confianza al } 95 \\
\% \text { para la diferencia(a) }\end{array}$} \\
\hline & & & & $\begin{array}{l}\text { Límite } \\
\text { superior }\end{array}$ & $\begin{array}{l}\text { Límite } \\
\text { inferior }\end{array}$ \\
\hline $0-3.99$ & $1,014^{*}$ & , 135 & ,000 & $-1,282$ &,- 747 \\
\hline $4-4.99$ & $0,816^{*}$ &, 118 & ,000 & $-1,049$ &,- 583 \\
\hline $5-5.99$ & $0,334^{*}$ &, 125 & ,009 &,- 583 &,- 086 \\
\hline $6-6.99$ & ,257 &, 143 &, 074 &,- 540 & ,025 \\
\hline $7-7.99$ & $-0,329 *$ & , 139 & .019 & ,055 & ,604 \\
\hline $8-8.99$ & $-0,805^{*}$ &, 147 &, 000 &, 514 & 1,096 \\
\hline $9-10$ & $-1,031 *$ & , 186 & ,000 & ,662 & 1,400 \\
\hline
\end{tabular}

Basadas en las medias marginales estimadas. * La diferencia de las medias es significativa al nivel , 05.

a ajuste para comparaciones múltiples: Bonferroni.

Como podemos observar en el Gráfico 1, a medida que los alumnos obtienen menor calificación en su examen final, el sistema de evaluación acumulativa por actividades mejora su resultado final. Sin embargo, la diferencia entre la calificación del examen final y la forma de evaluación llevada a cabo se incrementa a mayor calificación del examen.

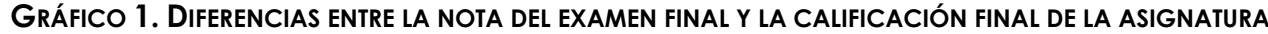

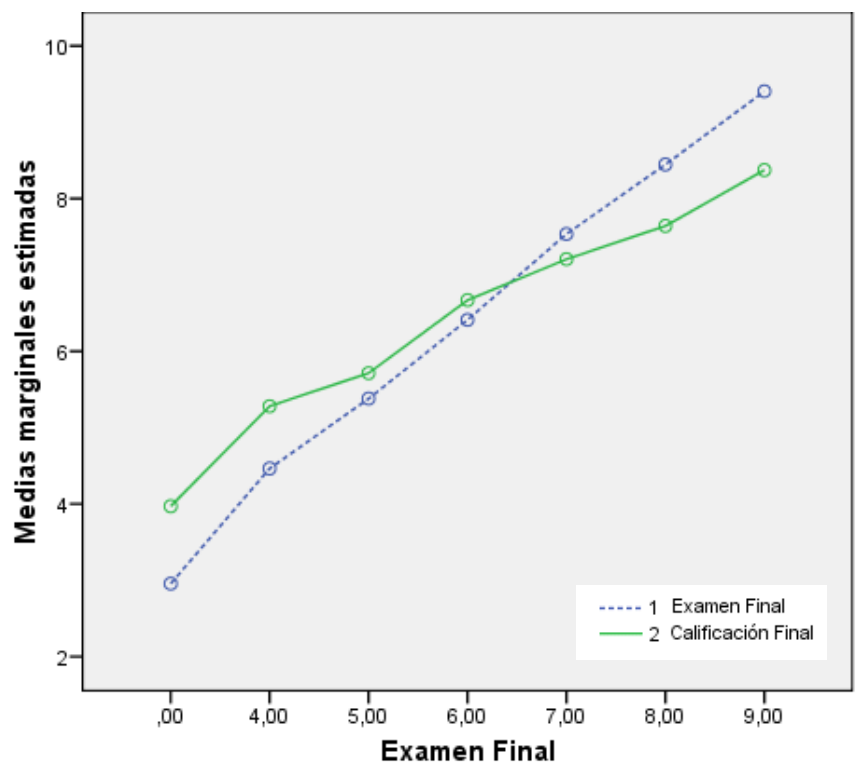

educade, nㅇ 2, 2011, p. 75 
Si comprobamos estos datos analizando los resultados de cada uno de los tres profesores de la asignatura que han participado en el proceso (Grafico 2), podemos observar cómo, a pesar de ligeras diferencias en la puntuación, la tendencia en los resultados en la evaluación de los alumnos se mantiene. Resulta interesante destacar que la nota de cambio en la tendencia varía según el profesor.

\section{Gráfico 2. Diferencias entre la nota del examen final y la Calificación FINAL DE LA ASIGNATURA POR PROFESOR}
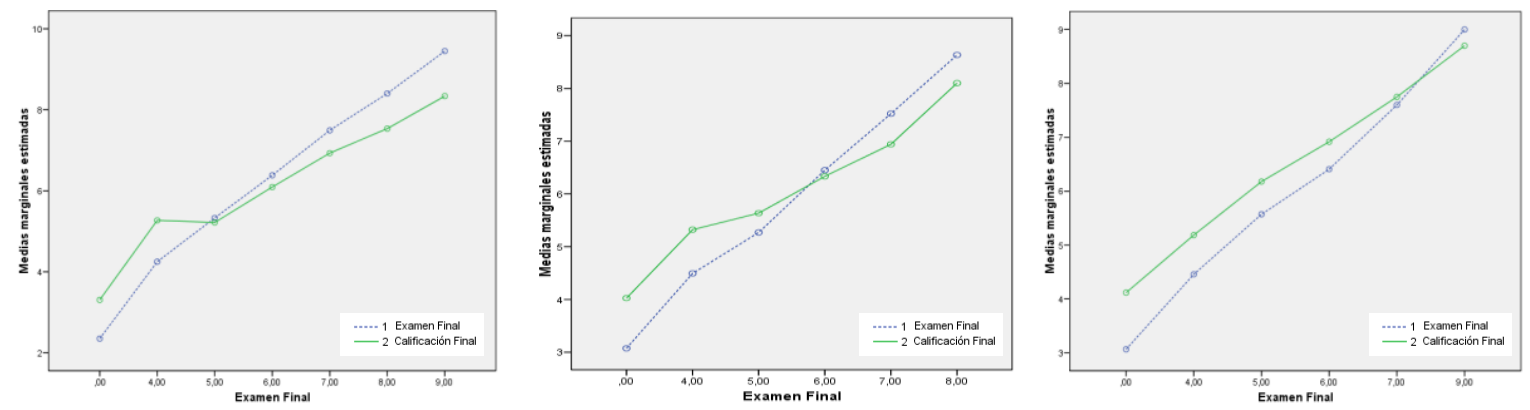

Si, por otro lado, comprobamos la correlación existente entre los resultados obtenidos por los alumnos, tanto en la calificación final de la asignatura como en los exámenes realizados con las características demográficas de los participantes, obtenemos que tan sólo existe correlación positiva, estadísticamente significativa, entre los resultados en los exámenes, tanto segundo parcial como final, con la nota obtenida en el examen de selectividad.

Finalmente observamos las diferencias en las puntuaciones obtenidas tanto en el examen final como en la calificación global de la asignatura respecto a la motivación que ha llevado a los alumnos a seleccionar el Grado en el que se han matriculado. Si bien es cierto que los resultados son meramente descriptivos y no tiene sentido analizar si existen diferencias entre medias, debido a las diferencias en el tamaño de los grupos, podemos plantear algunas reflexiones. Si tenemos en cuenta las calificaciones medias para el conjunto de los alumnos podemos observar como aquellos que indican haber seleccionado su carrera universitaria porque "les gustaba la economía previamente", son los que mejor calificación obtienen (Gráfico 3).

Gráfico 3. Diferencias entre la nOta del EXAMEN final y LA CALIFICACIÓN FINAL DE LA ASIGNATURA POR MOTIVACIÓN
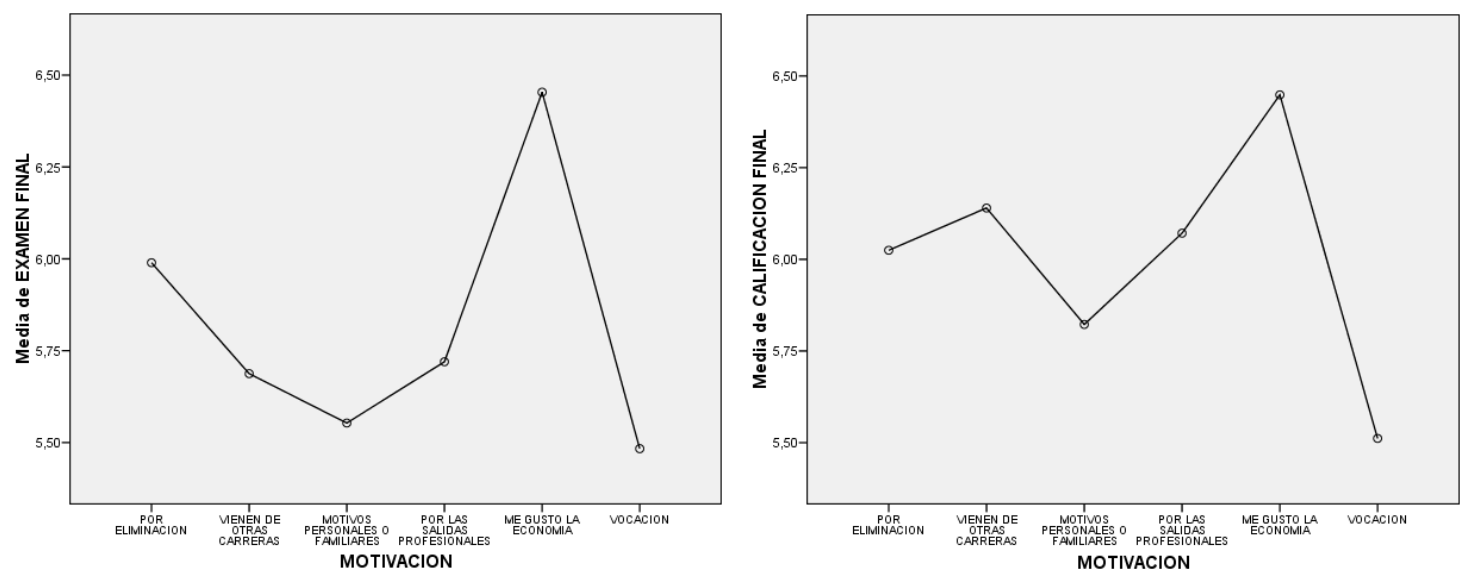
Ahora bien, para averiguar si los estudiantes que no habían superado el examen final condicionaban los resultados obtenidos, se limitaron los análisis a aquellos que habían obtenido una calificación en el examen final superior o igual a 5 puntos sobre 10. Efectivamente existen diferencias ya que los alumnos que proceden de otras carreras son, en este caso, los que consiguen mejores resultados tanto en el examen final como en la calificación final de la asignatura (Gráfico 4).

\section{GRÁFICO 4. DifERENCIAS ENTRE LA NOTA DEL EXAMEN FINAL Y LA CALIFICACIÓN FINAL DE LA ASIGNATURA POR MOTIVACIÓN}

(Nota del examen final $\geq 5$ )
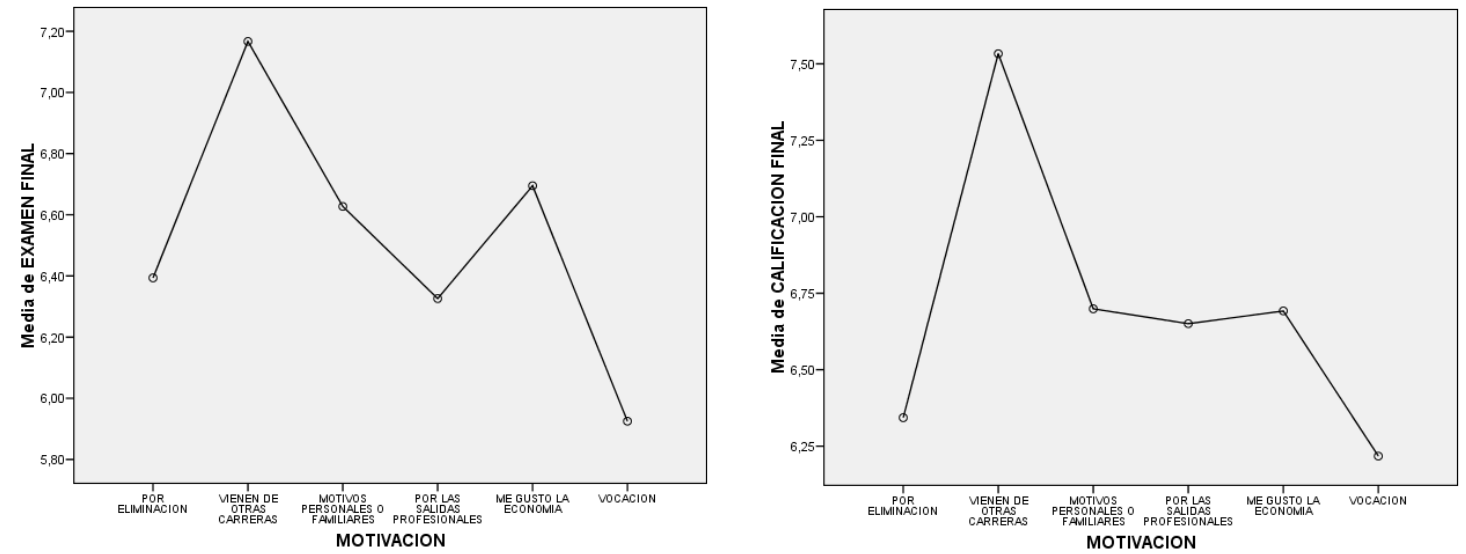

Por otro lado, se repitieron los análisis limitando tan solo a aquellos alumnos que han obtenido una calificación final igual o superior a 5 en la asignatura, los resultados no varían respecto a los análisis anteriores (Gráfico 5).

\section{GrÁfico 5. Diferencias ENTRE LA NOTA DEL EXAMEN FINAL Y LA CALIFICACIÓN FINAL DE LA ASIGNATURA POR MOTIVACIÓN}

(Calificación final de la asignatura $\geq 5$ )
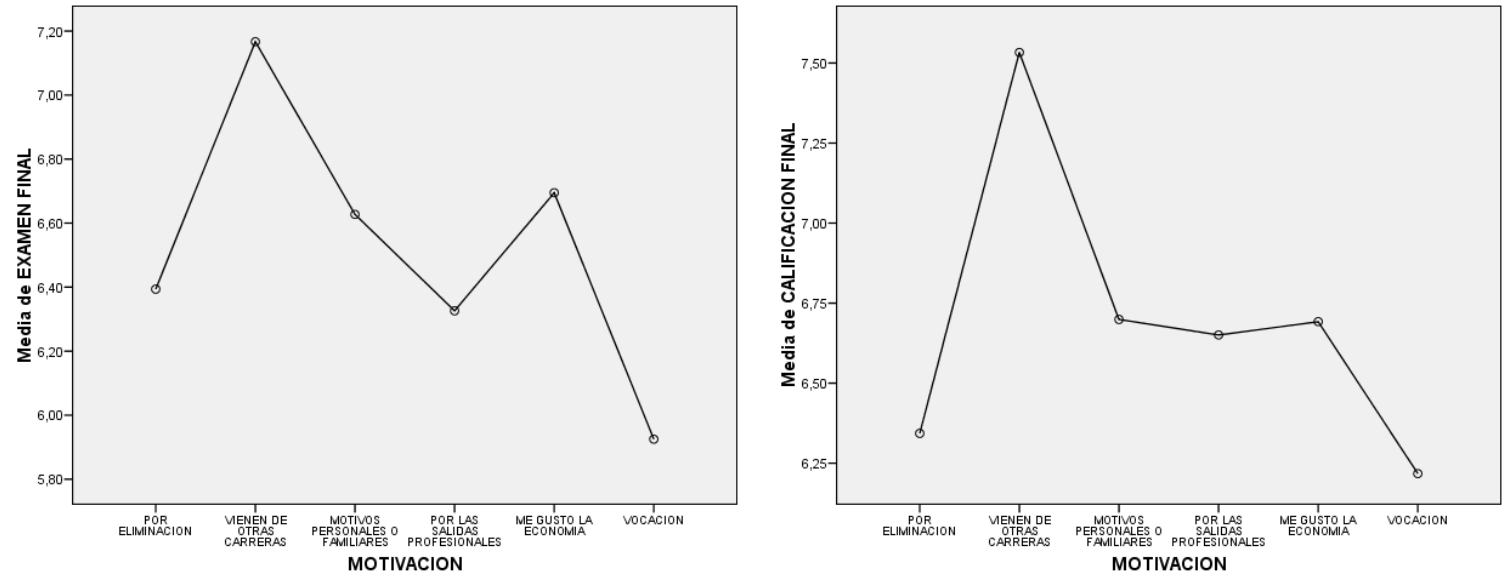


\section{DISCUSIÓN Y CONCLUSIONES}

Muchos estudios avalan la interrelación entre la metodología docente y la calidad del aprendizaje. Sin embargo, el aprendizaje y la evaluación académica también están íntimamente relacionados ya que el tipo de valoración condiciona el proceso y la calidad del aprendizaje. El alumno va a estudiar en función de cómo y qué evalúa el profesor. Con una metodología basada en la clase magistral, la evaluación se fundamenta en la nota del examen final. Sin embargo, la metodología activa en la implantación de los nuevos Grados en el marco del EEES requiere un cambio en el proceso de valoración en la adquisición de competencias y conocimientos de los estudiantes. Un criterio de evaluación de la metodología activa es la evaluación acumulativa por actividades, es decir, valorar todas las actividades que realiza el alumno en su proceso de aprendizaje.

Este sistema de calificación trata de evaluar el proceso de aprendizaje en su totalidad y tiene como uno de sus objetivos principales que los alumnos trabajen diariamente y participen activamente en la asignatura, siendo responsables de su propio aprendizaje. Además, la materia de contabilidad financiera requiere una progresiva y continuada adquisición de conocimientos ya que se necesita ir comprendiendo la materia previa para poder avanzar en el aprendizaje con lo que este nuevo sistema debería favorecer dicho proceso.

La evaluación acumulativa por actividades, en el marco del nuevo EEES, facilita la evaluación por parte del profesor de ciertas competencias de los alumnos y no sólo sus conocimientos teóricos de la asignatura. Estas competencias serán tan necesarias o más en el momento que los alumnos comiencen su trayectoria profesional que los conocimientos teóricos. Por eso el cambio en el sistema de evaluación que ha supuesto la implantación de la nueva metodología de enseñanza activa ha supuesto una gran oportunidad tanto para profesores como para los alumnos de conseguir que la formación universitaria se aproxime un poco más a la situación real en el mundo laboral. Sin embargo, los resultados obtenidos en este trabajo muestran como la evaluación acumulativa por actividades, cuya implementación trata de solucionar las deficiencias que existen en la evaluación tradicional basada exclusivamente en un examen final y que en muchos casos no reflejaba la comprensión real de la asignatura por parte del alumno, también presenta cierto tipo de limitaciones.

Los alumnos que sacan mejores notas en el examen final, por lo general, son aquellos que más conocimientos han adquirido de la materia. Esto no implica que sean los que mejor los apliquen posteriormente a la vida real, de ahí que sea positivo un sistema de evaluación en el que se evalúen competencias y no sólo conocimientos, pero sí que es claro que son los que más conocimientos teóricos, o en el caso de la asignatura de contabilidad financiera teórico-prácticos, han adquirido. Los resultados obtenidos en nuestro trabajo parecen indicar que el sistema de evaluación acumulativa por actividades propuesto con los nuevos Grados no les permite obtener a los estudiantes que obtienen las mejores calificaciones en el examen final (por tanto un mayor nivel de conocimientos) una buena calificación si no han demostrado su aplicación a la práctica en los distintos seminarios. Sin embargo, todo parece indicar que el nuevo método de evaluación acumulativa por actividades presenta carencias y debería ser revisado.

Por otro lado, resulta interesante resaltar que la evaluación final de los estudiantes con mejores resultados en el examen final correlaciona significativamente con el examen parcial que incluía la mitad de la materia. Es decir, aquellos alumnos que obtuvieron las mejores notas en los parciales son los mismos, en términos generales, que los que mejor resultado obtienen en el examen final. Esto se explica porque los alumnos que han ido superando los parciales han estudiado de manera continuada durante todo el 
curso, es decir, han asimilado los contenidos teóricos de contabilidad financiera y han sabido aplicarlos en la práctica. También existe una alta correlación positiva entre los resultados anteriores con la nota que los alumnos han obtenido en selectividad. De hecho si se considera la nota de acceso a la universidad como una medida de capacidad intelectual, los buenos alumnos continúan con la tendencia de buenos resultados en los exámenes. Además, todo parece indicar que aquellos alumnos que declaran haber escogido la carrera "porque les gustaba la economía previamente", obtienen unos resultados mejores y más homogéneos que el resto de alumnos cuyas motivaciones eran otras.

Por lo tanto y a partir de los resultados obtenidos en este trabajo, se pone en duda el sistema de evaluación recogido en los planes de estudios aprobados por la Agencia Nacional de Evaluación de la Calidad y Acreditación (ANECA) para los estudios de GADE y GECO tal y como se implementa en la realidad de la materia considerada. A pesar de todo, en conjunto, el aprendizaje de los alumnos creemos que ha sido más significativo, si bien hay que volver a testar el conocimiento de la materia a largo plazo. Al tratarse de un experimento diseñado a medida, con una base de datos propia, este trabajo aporta un valor añadido a posibles investigaciones en otras universidades o en otras disciplinas.

Por todo lo expuesto anteriormente, entendemos que estos resultados deben dar lugar a ampliar el experimento, ya que de darse unos resultados similares, se deberían producir cambios significativos en este sistema de evaluación. Una posible solución a la problemática expuesta sería que el alumno, en función de sus capacidades, motivación y modo de estudio, pudiera elegir entre un examen final del curso y una evaluación acumulada por actividades. Sin embargo, habría que analizar en profundidad dónde reside el problema detectado en el sistema de evaluación acumulativa por actividades y tratar de proponer soluciones, ya que consideramos que el desarrollo y evaluación de las competencias es algo muy positivo para los alumnos. Otra propuesta podría ser la elaboración de portfolios digitales en las que el alumno se autoevalúa y después es evaluado por pares y por el profesor. Todo ello en la línea de promover una mejora continua en la docencia universitaria y un aprendizaje significativo del alumno.

\section{BIBLIOGRAFÍA}

AHUMADA ACEBEDO, P. [1998]: Hacia una evaluación de los aprendizajes en una perspectiva constructivista, Revista de Enfoques Educacionales, 1 (2): 1-16. http://www.saladehistoria.com/educacion/Ahumada_1998.pdf

AlbaneSE, M.A. Y MitChelL, S. [1993]: Problem-based learning: A review of literature on its outcomes and implementation issues, Academic Medicine, 68: 52-81.

Álvarez VALDIVIA, I. [2008]: Evaluación del aprendizaje en la universidad: una mirada retrospectiva y prospectiva desde la divulgación científica, Revista Electrónica de Investigación Psicoeducativa, 14 (6): 235-272.

ANTEPOHL, W. Y HERZIG, S. [2002]: Problem-based learning versus lecture-based learning in a course of basic Pharmacology: a controlled, randomized study. Medical Education, 33 (2): 106-113.

ARQUERO J.L, JIMÉNEZ, S.M Y JOYCE, J. [2004]: Skills development, motivation and learning in financial statement analysis: an evaluation of alternative types of case studies, Accounting Education, 13 (2): 191-212.

ARQUERO, J.L., HASSALL, T., JOYCE, J. Y DONOSO, J.A. [2007]: Accounting students and communication apprehension: A study of Spanish and UK students, European Accounting Review, 16 (2): 299-322. 
AsBAUGH, H., JOHNSTONE, K.M. y WARFIELD, T.D. [2002]: Outcome assessment of a writing-skill improvement initiative: results and methodological implications, Issues in Accounting Education, 17 (2): 123-148.

BALLANTINE, J.A., Y MCCOURT, P. [2004]: A critical analysis of student's perceptions of the usefulness of the case study method in an advanced management accounting module: the impact of relevant work experience, Accounting Education, 13 (2): 171-189.

Ballantine, J.A., DufF, A. Y MCCOuRT, P. [2008]: Accounting and business students' approaches to learning: A longitudinal study, Journal of Accounting Education, 26 (4): 188-201.

BONK, C., SMITH, J. y SteVEnSON, G. [1998]: Alternative instructional strategies for creative and critical thinking in the Accounting curriculum, Journal of Accounting Education, 16 (2): 261-293.

Boyce, G., Williams, S., Kelly, A. Y YeE, H. [2001]: Fostering deep and elaborative learning and generic (soft) skill development: the strategic use of case studies in accounting education, Accounting Education, 10 (1): 37-60.

BRYANT, S.M. y HUNTON, J.E. [2000]: The use of technology in the delivery of instruction: Implications for Accounting Educators and Education Researchers, Issues in Accounting Education, 15 (1): 129-162.

CAMACHO MIÑANO, M.M. [2009]: Uso de mandos interactivos en la docencia universitaria. Aplicación práctica en la asignatura de Contabilidad Financiera I. Proyecto MATHEMATICAL-LEARNING. http://cimanet.voc.edu/mel/content/view/75/82/lang,spanish/

Chaín NavarRo, C.; Martínez Solís, L. y SÁnchez Baena, J.J. [2010]: Motivar desde la innovación en la enseñanza universitaria: el Blog Qalidad, Revista de Educación a Distancia, 21:1-17

CLINTON, B.D. Y KOHLMEYER III, J.M. [2005]: The effects of group quizzes on performance and motivation to learn: two experiments in cooperative learning, Journal of Accounting Education, 23: 96-116.

CULLEN, J., RICHARDSON, S. y O'BRIEN, R. [2004]: Exploring the teaching potential of empirically-based case studies, Accounting Education, 13 (2): 251-266.

DANZINGER, N. Y EDEN, Y. [2006]: Student career aspirations and perceptions: The case of Israeli Accounting students, Accounting Education: an International Journal, 15(2): 113-134.

Delgado García, A.M. y Oliver Cuello, R. [2006]: La evaluación continua en un nuevo escenario docente, Revista de Universidad y Sociedad del Conocimiento, 3 81): 1-13. http://www.uoc.edu/ojs/index.php/rusc/article/view/v3nl-delgadooliver/v3n1-delgado-oliver

DOCHY, F.; SEGER, M. Y DIERICK, S. [2008]: Nuevas vías de aprendizaje y enseñanza: sus consecuencias: una nueva era de evaluación, Revista de Docencia Universitaria. 2 http://revistas.um.es/index.php/red_u/article/view/20051/19411

DuFF, A. [2004]: The role of cognitive learning styles in accounting education: developing learning competencies, Journal of Accounting Education, 22 (1): 29-52.

Del FAVERO, L.; BosCOlo, P.; VIDOTtO, G. y VICENTINI, M. [2007]: Classroom discussion and individual problem-solving in the teaching of History: Do different instructional approaches affect interest in different ways? Learning and Instruction, 17 (6): 635-657.

FELDER, R.M. [1995]: Cooperative learning in a sequence of engineering courses: a success story, Cooperative Learning and College Teaching Newsletter, 5 (2): $10-13$.

FELDER, R.M.; FELDER, G.N. y DietZ, E.J. [1998]: A longitudinal study of Engineering student performance and retention $v$. comparisons with traditionally-taught students, Journal of Engineering Education, 87 (4): 469-480. 
GABBIN, A.L. Y WOOD, L.I. [2008]: An experimental study of Accounting Majors' academic achievement using cooperative learning groups, Issues in Accounting Education, 23 (3): 391-404.

Gordon, J.A.; SHAFFer, D. W.; RAEMER, D. B.; PAWlowskl, J.; HuRford, W.E. y COOPER, J. B. [2006]: A randomized controlled trial of simulation-based teaching versus traditional instruction in medicine: a pilot study among clinical medical students, Advances in Health Sciences Education, 11 (1): 33-39.

GREenHOW, C.; RobelIA B. Y HUGHES, J.E. [2009]: Learning, teaching and scholarship in a digital age. Web 2.0 and classroom research: what path should we take now?, Educational Researcher, 38 (4): 246-259.

GURPINAR, E.; MUSAL, B.; AKSAKOGLU, G. Y UCKU, R. [2005]: Comparison of knowledge scores of medical students in problem-based learning and traditional curriculum on public health topics, BMC Medical Education, 5 (7): 1-8.

HAGHIGHI, A.M.; VAKIL, R. Y WETIBA, J.K. [2006]: Reverse-Traditional/Hands-on: An alternative method of teaching Statistics, Applications and Applied Mathematics, 1 (1): 62-82.

Halabl, A.K.; TuOVINen, J.E. y Farley, A.A. [2005]: Empirical evidence on the relative efficiency of worked examples versus problem-solving exercises in Accounting Principles instruction, Issues in Accounting Education, 20 (1): 21-32.

HARRINGTON, K. [2010]: Assessment: How do you really know? in UPTON, D. y TRAPP, A. (eds) Teaching Psychology in Higher Education (Blackwell and British Psychological Society Publishing.).

HeAGY, C.D. Y LeHMANN, C.M. [2005]: "Is PBL an improved delivery method for the Accounting curriculum?" Advances in Accounting Education, 7, 225-251.

HOLBERT, K.E. y KARADY, G.G. [2009]: Strategies, Challenges and prospects for active learning in the computer-based classroom, IIIE Transactions on Education, 52 (2): 31-38.

JOHNSON, H.D. Y DASGUPTA, N. [2005]: Traditional versus Non-traditional teaching: Perspectives of students in Introductory Statistics Classes, Journal of Statistics Education, 13(2). http://www.amstat.org/publications/JSE/v13n2/johnson.html.

KVAM, P.H. [2000]: The effect of Active Learning methods on student retention in Engineering Statistics, The American Statistician, 54 (2): 136-140.

LORD, B.R. Y ROBERTSON, J. [2006]: Students' experiences of learning in a third-year management accounting class: evidence from New Zealand, Accounting Education: An International Journal, 15 (1): 41-59.

LYCKE, K.H.; GROTTUM, P. Y STROMSO, H.I. [2006]: Student learning strategies, mental models and learning outcomes in problem-based and traditional curricula in medicine, Medical Teacher, 28 (8): 717-722.

MARTIN, T.; RIVALE, S.D. Y DILLER, K.R. [2007]: Comparison of student learning in challengebased and traditional instruction in Biomedical Engineering, Annals of Biomedical Engineering, 35(8): 312-323.

MARTON, F. Y SÄLJÖ, R. [1976]: On qualitative differences in learning: I. Outcome and process, British Journal of Educational Psychology, 46 (1): 115-127.

MILNE, M. J. Y MCCONNEILL, P.J. [2001]: Problem-Based learning: a pedagogy for using case material in accounting education, Accounting Education, 10(1): 61-82.

MOLERO LÓPEZ BARAJAS, D. [2007]: Rendimiento académico y opinión sobre la docencia del alumnado participante en experiencias piloto de implantación del Espacio Europeo de Educación Superior, Relieve, 13 (2). http://www.uv.es/RELIEVE/v13n2/RELIEVEv13n2_2.pdf

MURPHY, R. [2006]: Evaluating new priorities for assessment in higher education en Bryan, C. y Clegg, K. (eds.): Innovative assessment in Higher education. (New York: Routledge). 
NANDI, P.L.; CHAN, J.N.F. Y CHAN, C.P. [2000]: Undergraduate medical education: comparison of problem-based learning and conventional teaching. Hong Kong Medical Journal, 6: 301-306.

NouRI, H. Y SHAHID, A. [2005]: The effect of Power Point presentations on student learning and attitudes, Global Perspectives on Accounting Education, 2: 53-73. http://gpae.bryant.edu/ gpae/vol2/04-

046\%20The\%20Effect\%20of\%20PowerPoint\%20Presentations\%20on\%20Student\% 20Learning.pdf

POTTER, B.N. Y JOHNSTON, C.G. [2006]: The effect of interactive on-line learning systems on student learning outcomes in accounting, Journal of Accounting Education, 24 (1): 16-34.

PRINCE, M.J. [2004]: Does active learning work? A review of the research, Journal of Engineering Education, 93 (3): 223-246.

PRINCE, M.J. Y FELDER, R.M [2006]: Inductive teaching and learning methods: definitions, comparisons and research bases, Journal of Engineering Education, 95 (2): 123-138.

RAVENSCROFT, S.P.; BUCKLESS, F.A.; MCCOMBS G.B. y ZUCKERMAN, G.J. [1995]: Incentives in student team learning: An experiment in cooperative group learning, Issues in Accounting Education, 10 (1): 97-109.

SÁNCHEZ , P. [2009]: The design, implementation and evaluation of a Pilot scheme adapted to the Bologna goals at tertiary level, Higher Education in Europe, 34 (1): 77-89.

SAVANDER-RANNE, C.; LUDEN, O.P. y Kolarl, S. [2008]: An alternative teaching method for electrical engineering courses, IEEE Transactions on Education, 51 (4): 423-431.

SCHMIDT, H.G.; DAPHNEE, W. D. Y PATEL, V.L. [1987]: Comparing the effects of problembased and conventional curricula in an international sample, Journal of Medical Education, 62 (4): 305-315.

SPRINGER, C.W. Y FAYE, A. [2004]: Business simulation to stage critical thinking in introductory accounting: rationale, design and implementation, Issues in Accounting Education, 19(3): 227-304.

TerenzinI, P.T.; CABrera, A.F.; ColbeCK, C.L.; PARente, J.M. y BJORKLUnd, S. A. [2001]: Collaborative Learning vs. Lecture/Discussion: Students' reported learning gains, Journal of Engineering Education, 90: 123-130.

TONG WU, Y.J.M. [2005]: An empirical test of cooperative learning in a passive learning environment, Issues in Accounting Education, 20 (2): 151-165.

TREES, A.R. Y JACKSON, M.H. [2007]: The learning environment in clicker classrooms: student processes of learning and involvement in large university-level courses using student response systems, Learning, Media and Technology, 32 (1): 21-40.

TOVIA, F. Y LIU, Y. [2007]: Students evaluating significant factors on retention: a statistical analysis, International Conference on Engineering Education- ICEE, September 3-7. Coimbra Portugal, http://icee2007.dei.uc.pt/proceedings/papers/123.pdf

UhARI, M., ReNKO, M. y SOINI, H. [2003]: Experiences of using an interactive audience response system in lectures, BMC Medical Education, 3 (12): 1-6.

URquía Grande, E.; Muñoz Colomina, C.I. y CANO MONTERO, E.I. [2009]: La simulación del cuadro de mando integral. Una herramienta de aprendizaje en la materia de contabilidad de gestión. Documento de trabajo n $465 / 2009$. Fundación de las Cajas de Ahorro, http://en.scientificcommons.org/51616205

VERIFICA UCM [2008]: Título de GADE y GECO. Madrid: ANECA.

VILLARDÓN, L. Y YÁNIZ, C. [2004]: El aprendizaje cooperativo y el nuevo concepto de evaluación,

http://webcache.googleusercontent.com/search?q=cache:3xW_alGONhEJ:gi ac.upc.es/pag/giac_cas/giac_jac/04/JAC04-

LV.htm+Zabalza,+M.+a.+(2001).+Evaluaci\%C3\%B3n+de+los+aprendizajes+en+l

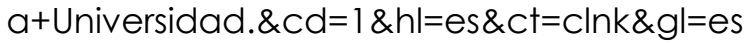


VYGOTSKY, L. [1962]: Thought and Language (Cambridge, Ma: MIT Press).

WeIL, S.; OYeleRE, P.; YeOH, J., Y FIRER, C. [2001]: A study of students perceptions of the usefulness of case studies for the development of finance and accounting related skills y knowledge, Accounting Education, 10(2): 123-146.

WOOTEN, T. C. [1998]: Factors influencing student learning in introductory Accounting Classes: a comparison of traditional and non-traditional students, Issues in Accounting Education, 13 (2): 357-373.

YURETICH, R.F.; KHAN, S.A.; LECKIE, R.M. Y CLEMENT, J.J. [2001]: Active-Learning methods to improve student performance y scientific interest in a large introductory Oceanography course, Journal of Geosciences Education, 49, (2): 111-119, http://www.geo.umass.edu/faculty/leckie/Yuretich\%20et\%20al.\%202001\%20JG E.pdf.

ZABALZA, M.A. [2001]: Evaluación de los aprendizajes en la Universidad. En García Valcárcel, A. Didáctica Universitaria (Madrid: La Muralla). 